\title{
Gephyrin Selective Intrabodies as a New Strategy for Studying Inhibitory Receptor Clustering
}

\author{
Paola Zacchi • Elena Dreosti • Michela Visintin • \\ Matteo Moretto-Zita • Ivan Marchionni • \\ Isabella Cannistraci - Zeynep Kasap • Heinrich Betz • \\ Antonino Cattaneo $\cdot$ Enrico Cherubini
}

Received: 20 September 2007 / Accepted: 9 October 2007 /Published online: 16 November 2007

(C) Humana Press Inc. 2007

\begin{abstract}
The microtubule-binding protein gephyrin is known to play a pivotal role in targeting and clustering postsynaptic inhibitory receptors. Here, the Intracellular Antibodies Capture Technology (IATC) was used to select two single-chain antibody fragments or intrabodies, which, fused to nuclear localization signals (NLS), were able to efficiently and selectively remove gephyrin from glycine receptor (GlyR) clusters. Co-transfection of NLS-tagged individual intrabodies with gephyrin-enhanced green fluorescent protein (EGFP) in HEK 293 cells revealed a partial relocalization of gephyrin aggregates onto the nucleus or in the perinuclear area. When expressed in cultured neurons,
\end{abstract}

Paola Zacchi, Elena Dreosti, and Michela Visintin contributed equally to this work.

P. Zacchi $(\square) \cdot$ E. Dreosti $\cdot$ M. Moretto-Zita $\cdot$ I. Marchionni $\cdot$

Z. Kasap - A. Cattaneo $\cdot$ E. Cherubini

Neuroscience Programme,

International School for Advanced Studies,

Via Beirut 2-4,

34014 Trieste, Italy

e-mail: zacchi@sissa.it

M. Visintin - I. Cannistraci - A. Cattaneo

Lay Line Genomics Spa,

Area Science Park,

34012 Basovizza, Trieste, Italy

H. Betz

Max-Planck Institute for Brain Research,

Deutschordenstrasse 46,

60528 Frankfurt am Main, Germany

Present address:

A. Cattaneo

European Brain Research Institute,

via del Fosso di Fiorano 64-65,

00143 Rome, Italy these intrabodies caused a significant reduction in the number of immunoreactive GlyR clusters, which was associated with a decrease in the peak amplitude of glycine-evoked whole cell currents as assessed with electrophysiological experiments. Hampering protein function at a posttranslational level may represent an attractive alternative for interfering with gephyrin function in a more spatially localized manner.

Keywords Gephyrin - Glycine receptor clusters · Intracellular antibodies · Hippocampal neurons · IATC

\section{Introduction}

At inhibitory synapses, the microtubule-binding protein gephyrin is known to play a pivotal role in targeting/ clustering postsynaptic inhibitory glycine receptors (GlyRs) (Feng et al. 1998) and $\gamma 2$-subunit containing gammaaminobutyric acid $\mathrm{A}\left(\mathrm{GABA}_{\mathrm{A}}\right)$ receptors (Kneussel et al. 1999). Gephyrin binds with high affinity to the $\beta$ subunit of the GlyR (Meyer et al. 1995), whereas it only functionally associates with $\mathrm{GABA}_{\mathrm{A}}$ receptor subtypes (Kirsch and Betz 1995; Meyer et al. 1995). Receptor anchoring is based on the interaction of gephyrin with the actin- and microtubulebased cytoskeleton (Kirsch 2006). GlyR clustering has been proposed to rely on the ability of gephyrin to multimerize into a submembraneous hexagonal protein lattice (Sola et al. 2004). The critical contribution of gephyrin to the postsynaptic localization of GlyRs in the spinal cord has been elegantly demonstrated by gene ablation experiments (Feng et al. 1998). Gephyrin knockout (KO) mice display a severe neuromotor phenotype, which is responsible for their death shortly after birth. The motor impairment observed in gephyrin-deficient mice has been mainly attributed to a 
severe deficit in glycinergic neurotransmission in the spinal cord (Feng et al. 1998). Interestingly, the complex motor disorder and neurologic symptoms observed in gephyrindeficient mice exhibit some phenotypic features of hyperexplexia in humans, suggesting the possible involvement of gephyrin in this disease.

Gephyrin KO mice have been extremely useful for unveiling several aspects of gephyrin-mediated receptor localization at central synapses (Feng et al. 1998). However, the lethality exhibited by these mice soon after birth represents an important limitation to their use as animal models for postnatal studies. To circumvent this problem, we have developed a more versatile and powerful molecular tool to achieve functional ablation of gephyrin based on intracellular expression of anti-gephyrin single-chain antibody fragments $(\mathrm{scFv})$ or intrabodies (Biocca et al. 1990). Unlike nucleic acid-based technologies, such as antisense (Kramer and Cohen 2004), zinc-finger proteins (Beerli and Barbas 2002), targeted gene disruption, or (ribonucleic acid) RNA interference (Tian et al. 2004), intrabodies operate at the posttranslational level, offering new experimental opportunities to analyze the function of a given molecule (Manikandan et al. 2007). Intrabodies can sterically prevent the interaction of their targets with other protein partners, or stabilize/destabilize them, thus preventing/facilitating their turnover and/or degradation. Finally, intrabodies fused to specific cellular localization sequences can target their antigens to specific subcellular compartments (Visintin et al. 2004a).

In the present study, a nuclear localization signal (NLS)targeted intrabody for the removal of endogenous gephyrin from inhibitory receptor clusters in cultured hippocampal neurons has been developed. As not all antibodies isolated by standard technologies fold well under conditions of intracellular expression (Visintin et al. 2004a), we exploited a yeast two-hybrid based intrabody selection technology, named Intracellular Antibody Capture Technique (IACT) (Visintin et al. 1999), to select anti-gephyrin intrabodies.

\section{Material and Methods}

\section{SPLINT Selection}

A Single Pot Library of INTrabodies (SPLINT) was used to isolate antibodies against ghephyrin (aa 153-348) bait. SPLINT contains genes encoding the heavy and light variable regions of the antibody producing cells (Visintin et al. 2004b). These are cloned in the IACT format as antibody fragments (scFv) (Visintin et al. 1999). The library design includes the capability to rapidly isolate soluble and stable scFvs directly from gene sequences with no handling of proteins.
A functional domain of the gephyrin protein (GDL aa 153-348) was cloned in the pMIC-BD1 vector and the expression of the fusion protein was assayed after the protein was extracted from the transformed yeast strain L40. The antigen was found to be expressed in optimum quantities in the yeast cells and did not transactivate the reporter genes (HIS3 and lacZ).

SPLINT library was then transformed into L40 yeast strain by using a rearranged lithium acetate transformation protocol. SPLINT and the ghephyrin bait were cotransformed into yeast cells as described (Visintin et al. 2002). Colonies were isolated on histidine-deficient plates and screened for $\beta$-gal activity on filters. Individual blue colonies were isolated by restreaking and again reassayed for $\beta$-gal activity. To isolate different anti-gephyrin scFvs, a colony-polymerase chain reaction (PCR) and BstNI fingerprinting analysis were performed and the isolated plasmids encoding anti-gephyrin scFvs were individually retested in a secondary IACT screening confirmed that true positives were identified that interact specifically with the original bait, but not with other lexA fusions (lexA-lamin).

\section{Cell Culture}

Primary cell cultures were prepared as previously described (Andjus et al. 1997). Briefly, 2- to 4-day-old (P2-P4) Wistar rats were decapitated after being anesthetized with an intraperitoneal injection of urethane $(2 \mathrm{mg} / \mathrm{kg})$. This procedure is in accordance with the regulation of the Italian Animal Welfare Act and was approved by the local authority veterinary service. Hippocampus was dissected free, sliced, and digested with trypsin, mechanically triturated, centrifuged twice at $40 \times g$. Dissociated neurons were plated in Petri dishes, and cultured for up to 14 days. Experiments were performed on cells cultured for at least 7 days. All the other cell lines used were routinely cultured at $37^{\circ} \mathrm{C}$ in Dulbecco's modified Eagle's Medium (DMEM) supplemented with $10 \%$ Fetal Bovine Serum (FBS) Penicillin $(100 \mathrm{U} / \mathrm{ml})$ and Streptomycin $(100 \mu \mathrm{g} / \mathrm{ml})$.

Immunofluorescence Staining

HEK 293 cells were fixed with 4\% $(w / v)$ paraformaldehyde $/ 4 \%$ sucrose for $15 \mathrm{~min}$, permeabilized with $0.1 \%(v / v)$ NP-40 for $5 \mathrm{~min}$, and then blocked with $10 \%(w / v)$ fetal bovine serum in phosphate-buffered saline (PBS) for $30 \mathrm{~min}$. Hippocampal neurons were fixed for $10 \mathrm{~min}$ with methanol at $-20^{\circ} \mathrm{C}$ or for 15 minutes, and processed as described above. Antibody staining was performed by incubation for 1 hour with primary antibodies and $45 \mathrm{~min}$ for secondary antibodies in blocking buffer. Enhanced green fluorescent protein (EGFP) was visualized by auto- 
fluorescence. Cluster density and brightness were analyzed with the Meta-Morph Imaging System (Universal Imaging, Westchester, PA). Twenty neurons were analyzed for each experiment (3-4 dendritic regions for each cell).

\section{Immunoprecipitation and Western Blot Analysis}

Transfections were performed with the standard calcium phosphate method. For gephyrin-EGFP and scFvGeph-2/ scFvGeph-9 coimmunoprecipitation HEK 293 cells overexpressing gephyrin-EGFP and scFvGeph-2/scFvGeph-9 were lysed in a lysis buffer containing $50 \mathrm{mM}$ Tris 7.5, $150 \mathrm{mM} \mathrm{NaCl}, 0.5 \% \mathrm{NP} 40,2 \mathrm{mM}$ ethylenediaminetetraacetic acid (EDTA) and 10\% glycerol and immunoprecipitated by the anti-SV5 antibody. Western blotting was performed according to standard procedures. Primary antibodies were revealed by HRPO-conjugated secondary antibodies (SIGMA), followed by enhanced chemiluminescence (Amersham).

\section{Electrophysiological Recordings}

Glycine- and $\alpha$-amino-3-hydroxy-5-methylisoxazole-4propionic acid (AMPA)-evoked currents were recorded at room temperature $\left(22-24^{\circ} \mathrm{C}\right)$ in the whole-cell configuration of the patch-clamp technique using a Multiclamp 700A (Axon Instruments, Foster City, CA, USA). Patch electrodes, formed from thin borosilicate glass (Hilgenberg, Malsfeld, Germany) had a resistance of 4-8 M $\Omega$ when filled with an intracellular solution containing: $\mathrm{CsCl}$ $137 \mathrm{mM}$, 4-2-hydroxyethyl-1-piperazineethanesulfonic acid (HEPES) $10 \mathrm{mM}$, BAPTA $11 \mathrm{mM}, \mathrm{MgCl}_{2} 2 \mathrm{mM}, \mathrm{Mg}$ ATP $2 \mathrm{mM}, \mathrm{CaCl}_{2} 1 \mathrm{mM}$. The composition of the external solution was: $\mathrm{NaCl} 137 \mathrm{mM}$, KCl $5 \mathrm{mM}, \mathrm{MgCl}_{2} 1 \mathrm{mM}$, $\mathrm{CaCl}_{2} 2 \mathrm{mM}$, glucose $20 \mathrm{mM}$, hemisodium HEPES $10 \mathrm{mM}$ (the $\mathrm{pH}$ was adjusted to 7.3-7.4 with $\mathrm{NaOH}$ ).

The stability of the patch was checked by repetitively monitoring the input and series resistance during the experiments. Cells exhibiting more than $15 \%$ changes were excluded from the analysis. Glycine- and AMPA-evoked currents were recorded at a holding potential of $-40 \mathrm{mV}$. Glycine and AMPA were applied through a multibarrel RSC-200 perfusion system (Bio-logic, Grenoble, France). With this system, a complete exchange of the solution around the cell was obtained in less than $30 \mathrm{~ms}$. In the case of glycine-evoked current, recordings were routinely performed in the presence of the $\mathrm{GABA}_{\mathrm{A}}$ receptor antagonist picrotoxin $(50 \mu \mathrm{M})$, the $\mathrm{GABA}_{\mathrm{B}}$ receptor antagonist CGP $55845(1 \mu \mathrm{M})$ and the broad spectrum ionotropic glutamate receptor antagonist kynurenic acid $(1 \mathrm{mM})$.

Drugs used were kynurenic acid, glycine, AMPA, strychnine all purchased from Sigma (Milan, Italy). Picrotoxin and CGP 55845 were purchased from Tocris
Cookson, Ltd. (Bristol, UK). Drugs were dissolved in water except CGP 55845 and picrotoxin that were dissolved in dimethylsulphoxide (DMSO) and ethanol, respectively. The final concentration of DMSO in the bathing solution was $0.1 \%$. At this concentration, DMSO alone did not modify the shape or the kinetics of the evoked currents. Currents were sampled at $20-100 \mathrm{kHz}$ and filtered with a cut-off frequency of $1 \mathrm{kHz}$. Data are expressed as mean \pm SEM. Statistical comparison was made using the unpaired $t$-test. $P<0.05$ was taken as significant.

\section{Results}

Selection of Two Gephyrin-Specific Intrabodies Using the IAC Technology

To select intracellular competent intrabodies by the twohybrid system, the linker region of gephryin (Fig. 1a) was cloned to the 3' of LexA (LexA-GLD; aa 153-348) and used to challenge a mouse SPLINT (Single Pot Library of INTracellular Antibodies) library of intrabodies (Visintin et al. 2004b). From the selection procedure a total of 90 colonies able to grow in the absence of histidine and showing activation of $\beta$-Galactosidase were obtained. The scFv-VP16 plasmids were isolated and sorted by their restriction patterns and sequences. The specificity of scFvs with different DNA fingerprints were reanalyzed using yeast strains expressing LexA-GLD and LexA-lamin, as nonrelevant antigen. Two different anti-gephyrin scFvs, designated scFvGeph-2 and scFvGeph-9, were thus identified. Analysis of the $\mathrm{V}$ region nucleotide sequences of the two selected anti-gephyrin intrabodies revealed that they were derived from germline $\mathrm{V}$ region genes with very few somatic mutations (data not shown). Both $\mathrm{scFv}$ shared the same $\mathrm{VH}$ region V11 that belongs to the subgroup VH-VIII, whereas VL regions belong to the kappa subgroup (kk4 and 19-32 for scFv2 and 9, respectively).

The selected intracellular antibodies were then expressed in mammalian cells, after subcloning of the $\mathrm{scFv}$ cassettes into the scFv-cyto-express plasmid (Persic et al. 1997). For intracellular detection, scFvs were equipped with the 11 amino acid long SV5 tag, preceded, in the nuclear tagged forms, by a tandem of three repeats of nuclear localization signals (NLS). Extracts from HEK 293 cells transiently transfected with scFvGeph-2 and scFvGeph-9 for different times demonstrated that the highest cytoplasmic expression was reached $24-30$ hours posttransfection. ScFvGeph-2 appeared to be more stable than scFvGeph-9, yielding higher expression levels in mammalian cells as compared to scFvGeph-9 (Fig. 1b). Similar results were obtained for the intrabodies provided of the NLS (data not shown). 


\section{a}
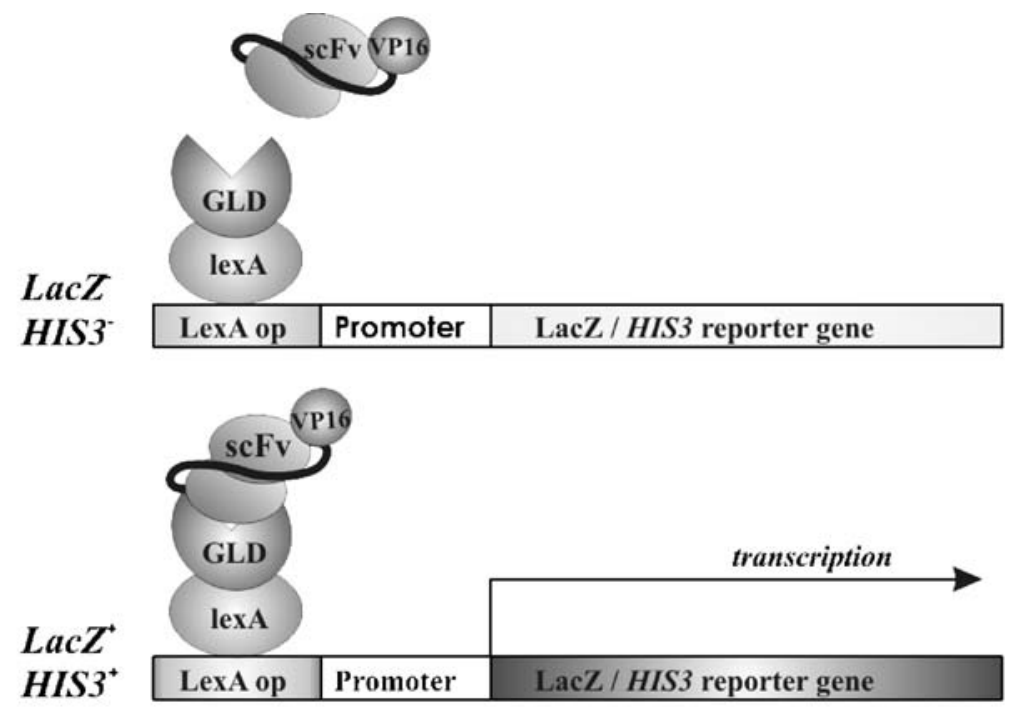

b

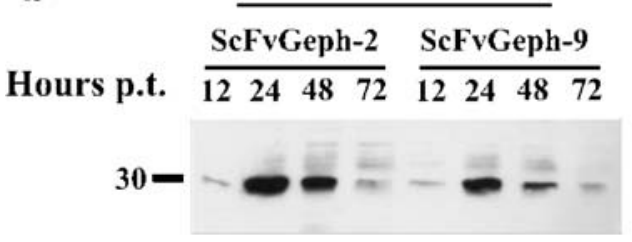

WB:anti-SV5

Figure 1 Isolation and characterization of $\mathrm{scFv}$ selected by IACTSPLINT technology. a. The scFv library is screened in yeast with the Gephyrin Linker Domain of (GLD) as a bait. Only those scFvs that retain the specific binding ability in vivo can activate the reporter genes, His 3 and LacZ. b. Western immunoblot using mAb anti-SV5 of

Gephyrin-Specific Intrabodies Recognize Gephyrin in Mammalian Cells

The ability of the selected intrabodies to recognize gephyrin in mammalian cells was assessed in immunocytochemical experiments performed on HEK 293 cells co-transfected with scFvGeph-2/scFvGeph-9 and gephyrin fused to EGFP. It is well-known that ectopically expressed gephyrin forms large intracytoplasmic aggregates characterized by their ability to actively sequester gephyrin interacting proteins (Kins et al. 2000; Meyer et al. 1995). Intrabodies expressed as leaderless cytoplasmic proteins showed a diffuse intracellular staining, typical of soluble cytoplasmic proteins (Fig. 2a). When gephyrin-EGFP and individual intrabodies were co-transfected, a massive fraction of scFvGeph-2/scFvGeph-9 was relocalized to gephyrin intracytoplasmic aggregates, thus resulting in colocalization of the two proteins (Fig. 2a). When the same experiments were performed using overexpression of the NLS-tagged intrabodies, a dramatic change in gephyrin distribution was observed (Fig. 2b). The efficient translocation of intrabodies into the nucleus because of the presence of the NLS cellular extracts of HEK 293 cells, transiently transfected with the indicated scFvs tagged with SV5. On the left, soluble fraction of scFvGeph-2 and scFvGeph-9 collected at different times after transfection, as indicated (Hours). On the right, insoluble fractions

analyzed as in the right panel

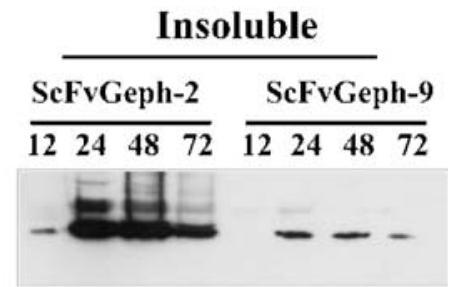

\section{WB:anti-SV5}

was associated with a partial reduction in the size of gephyrin cytoplasmic aggregates, most of them concentrated in the perinuclear area of the cell. The ability of scFvs to interact specifically with gephyrin was further analyzed by co-immunoprecipitation from extracts of HEK 293 cells coexpressing the two proteins. As shown in Fig. 2c, scFvGeph-2/scFvGeph-9 antibodies were able to co-immunoprecipitate gephyrin, suggesting that they interacted with gephyrin intracellularly.

\section{Gephyrin-Specific Intrabodies Alter Endogenous Glycine} Receptor Function

The ability of the anti-gephyrin intrabodies to bind and remove endogenous gephyrin from glycine receptor clusters was functionally assessed on cultured hippocampal neurons, known to highly express GlyRs (Danglot et al. 2004; Ito and Cherubini 1991). To this aim, NLS-tagged scFvGeph-2/ scFvGeph-9 were additionally equipped with EGFP tags to easily follow their fate within the transfected neurons. Twenty-four hours after transfection, immunocytochemical experiments revealed that not only gephyrin was 
Figure 2 Gephyrin-specific intrabodies interact with gephyrin in mammalian cells. a. Immunofluorescence assay of the subcellular distribution of SV5-tagged anti-gephyrin intrabody (scFv-Gephyrin) ectopically expressed in HEK 293 cells in single transfection experiment (left panel) and in co-transfection with gephyrinEGFP (right panels). ScFvGephyrin distribution was revealed with the anti-SV5 monoclonal antibody followed by anti-mouse TRITC-conjugated secondary antibody. Gephyrin distribution was revealed by the intrinsic green fluorescence of EGFP. b. Single (left panel) and double (right panels) transfection of the nuclear target NLS anti-Gephyrin intrabody ( $\mathrm{scFv}$ Gephyrin-NLS) was visualized as described in A. (Scale bar, $10 \mu \mathrm{m})$. c. Lysates of HEK 293 cells co-transfected with gephyrin-FLAG and scFvGeph-2 or scFvGeph-9 were immunoprecipitated with monoclonal antibodies anti-SV5 or anti-Myc as negative control. Immunoprecipitates were analyzed by western blotting using anti-FLAG and anti-SV5 antibodies, as indicated a
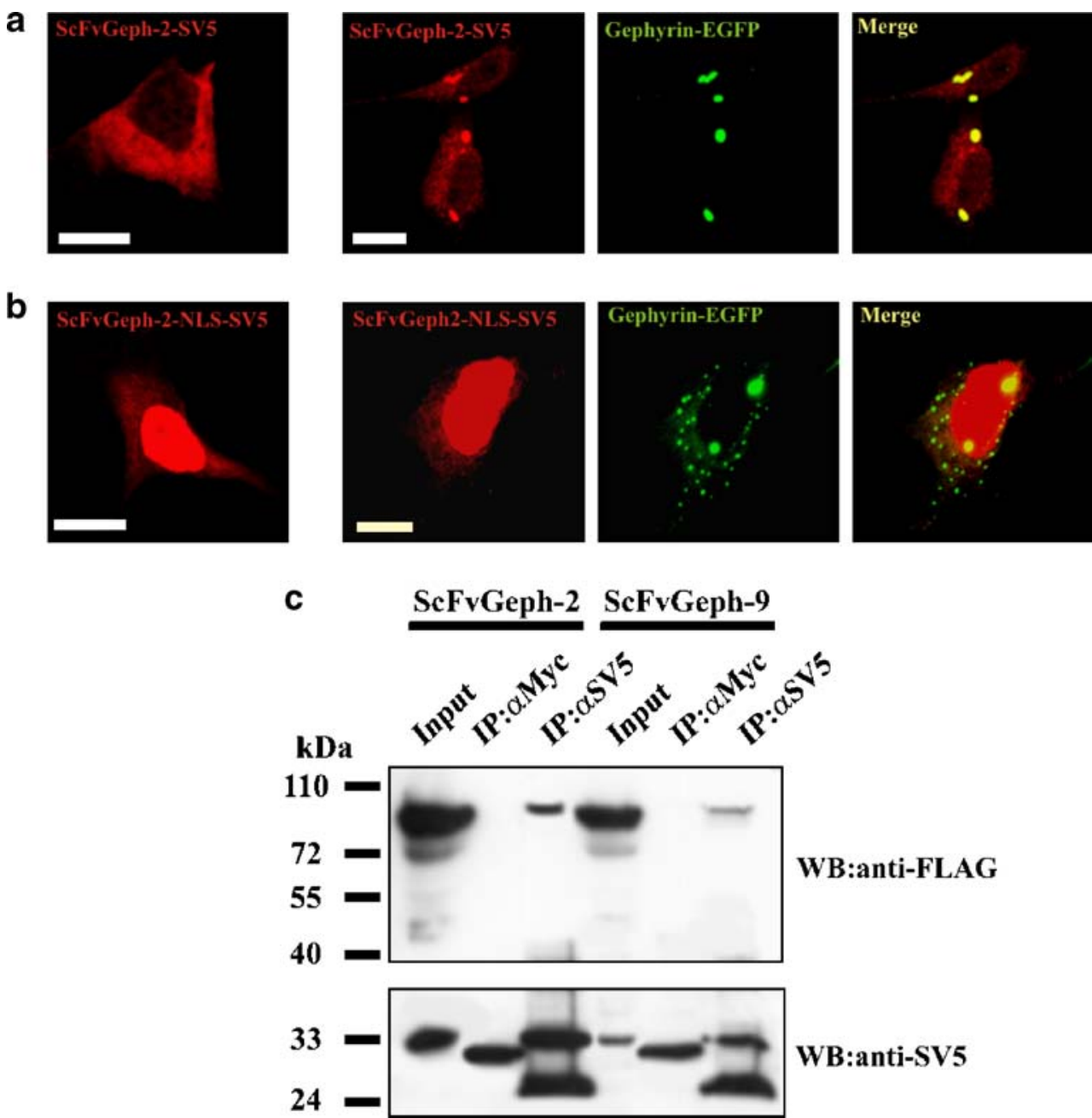

efficiently removed from most subsynaptic sites (Fig. 3a), but also that the number of GlyR clusters were dramatically reduced (Fig. 3b) compared to neurons transfected with EGFP alone. A quantitative analysis of immunoreactive gephyrin puncta in scFv-gephyrin-NLS transfected neurons revealed that in comparison to cells transfected only with EGFP, cluster fluorescence was significantly reduced. Fluorescence intensity values per square micron $\left(\mu \mathrm{m}^{2}\right)$ of dendritic surface were $2,857 \pm 500$ and $1,205 \pm 134$ in controls and scFv-gephyrin-NLS transfected cells, respectively (20 cells, detected in four different experiments, Fig. 3c). Similar results were found for GlyRs (fluorescence intensity values per square micron of dendritic surface were $2,211 \pm 378$ and $1,152 \pm 72$ in controls and in scFv-gephyrinNLS transfected cells, respectively; $n=20$ in both cases; Fig. 3c).

Glycine receptor responses in NLS-EGFP-tagged scFvGeph-2 transfected neurons were analyzed in electrophysiological experiments, using the whole-cell configuration of the patch clamp technique. Recordings were routinely performed in the presence of the GABAA receptor antagonist picrotoxin $(50 \mu \mathrm{M})$, the $\mathrm{GABA}_{\mathrm{B}}$ receptor antagonist
CGP $55845(1 \mu \mathrm{M})$, and the broad spectrum ionotropic glutamate receptor antagonist kynurenic acid $(1 \mathrm{mM})$. All cells tested $(n=99)$ responded to glycine application with inward currents of variable amplitude. These were readily blocked by the selective glycine receptor antagonist strychnine $(0.5 \mu \mathrm{M})$, indicating that they were mediated by glycine receptors (data not shown). As shown in Fig. 4a, glycine-evoked currents from transfected cells were significantly reduced in amplitude as compared to controls (nontransfected cells from the same dish) or to hippocampal neurons transfected only with EGFP. Glycine $(0.6 \mathrm{mM})$ induced current responses whose peak amplitude was $0.75 \pm$ $0.11 \mathrm{nA}(n=11), 1.52 \pm 0.12 \mathrm{nA}(n=9)$, and $1.6 \pm 0.16 \mathrm{nA}$ $(n=9)$ in NLS-scFvGeph-2, EGFP and nontransfected cells, respectively. The value obtained in transfected cells was significantly different from that detected in EGFP positive cells $(p<0.01)$ or in nontransfected neurons $(p<$ 0.01). In comparison with nontransfected or EGFP-transfected cells, NLS-scFvGeph-2-transfected neurons exhibited a concentration-response curve for glycine that was compressed downward and to the right (Fig. 4b). $\mathrm{EC}_{50}$ values were $254 \mu \mathrm{M}$ and $125 \mu \mathrm{M}$ for NLS-scFvGeph-2- and 
a
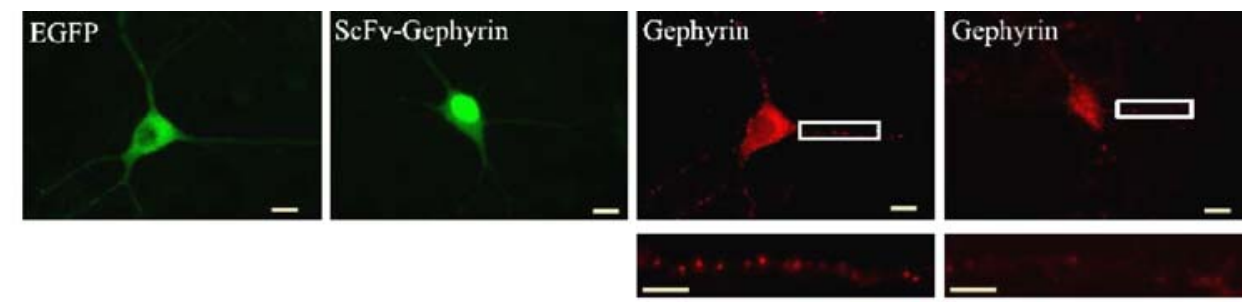

b
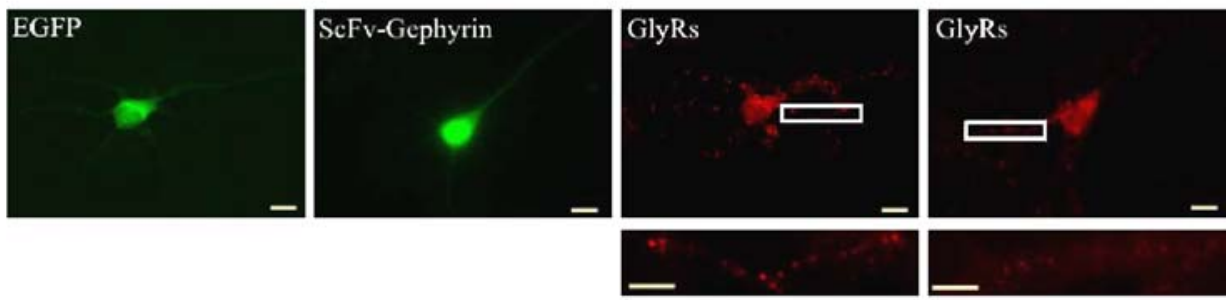

C

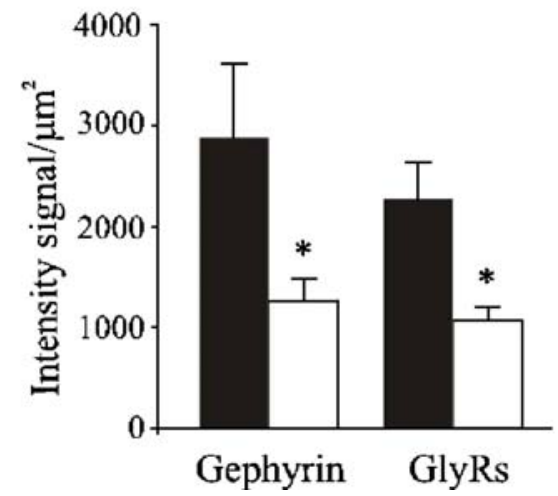

Figure 3 ScFv-Geph2-EGFP-NLS transfected in hippocampal neurons displaces gephyrin from its subsynaptic sites. A, B. Hippocampal neurons transfected with EGFP as negative control $(E G F P)$ and with scFv-Geph-2-EGFP-NLS ( $s c F v$-Geohyrin). Endogenous gephyrin (a) and GlyR (b) distributions were revealed using the monoclonal antibodies mAb7a (for gephyrin) and mAb4a (for GlyR) followed by anti-mouse TRITC-conjugated secondary antibody (Invitrogen). Note that the nuclear accumulation of scFv-Gephyrin-NLS was associated

EGFP-transfected cells, respectively. In contrast, current responses elicited in the same neurons by $\alpha$-amino-3hydroxy-5-methylisoxazole-4 propionic acid (AMPA $50 \mu \mathrm{M}$, in the presence of tetrodotoxin [TTX] $1 \mu \mathrm{M})$ were similar in amplitude $(0.20 \pm 0.03 \mathrm{nA}, n=11$ and $0.21 \pm$ $0.02 \mathrm{nA}, n=11, p>0.5$; Fig. $4 \mathrm{a}$ ) in both NLS-scFvGeph-2and EGFP-transfected cells, respectively, indicating that gephyrin intrabodies selectively reduce surface glycine receptor numbers.

\section{Discussion}

The main goal of the present study was to select competent cytoplasmic intrabodies against the scaffolding molecule gephyrin to use as a molecular tool to knock out gephyrin function in vivo. This strategy is of particular relevance as disrupting gephyrin gene in knockout mice leads to a lethal phenotype, thus hindering the study of gephyrin functions with a marked reduction of both gephyrin $(\mathrm{scFv}+$ Gephyrin in $\mathrm{A})$ and glycine receptor clusters $(\mathrm{scFv}+\mathrm{GlyRs}$ in $\mathrm{B})$. Bottom panels represent enlargements of the boxed areas. Scale bars: $10 \mu \mathrm{m}$. c. Quantitative analysis of cluster fluorescence on hippocampal neurons transfected with EGFP and scFv-Gephyrin-NLS. Each column represents the intensity signal normalized to the unit area (in $\mu \mathrm{m}^{2}$ ) of dendritic surface ( 20 cells in each column) in control conditions (black) and in the presence of scFv-Gephyrin-NLS (white); ${ }^{*} p<0.05$

at later stages of postnatal development. To exploit the intrabodies-based retargeting scheme for depleting endogenous gephyrin from inhibitory synapses, the linker or intervening region of gephyrin molecule was used for the two-hybrid selection method. This domain was chosen as it is not engaged in gephyrin oligomerization, a process regarded as the molecular mechanism responsible for inhibitory receptors accumulation at postsynaptic sites (Sola et al. 2004). As a consequence, the selected intrabodies should recognize gephyrin upon its self-assembly into the submembraneous protein lattice underlying inhibitory receptor clusters. Several lines of evidence support that the selected intrabodies are fully functional inside the cell. First, both intrabodies immunoprecipitate gephyrin upon their co-expression in HEK 293 cells. Second, they are specifically relocalized to gephyrin cytoplasmic aggregates. Interestingly, in the NLS-tagged format to divert intrabodies localization, they were able to promote a partial relocalization of gephyrin aggregates into the nucleus. We believe 
a

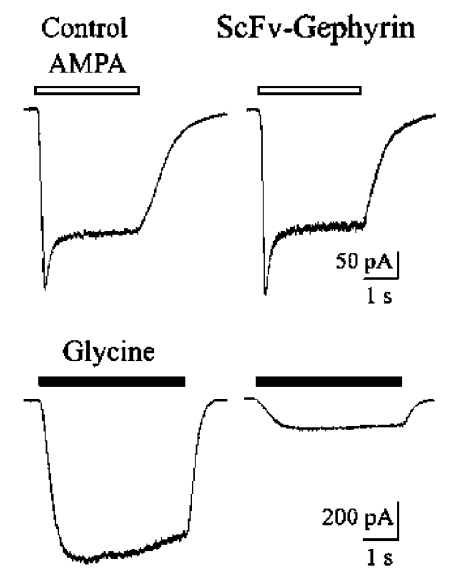

b

\section{Control}

- ScFv-Gephyrin

$\triangle$ EGFP

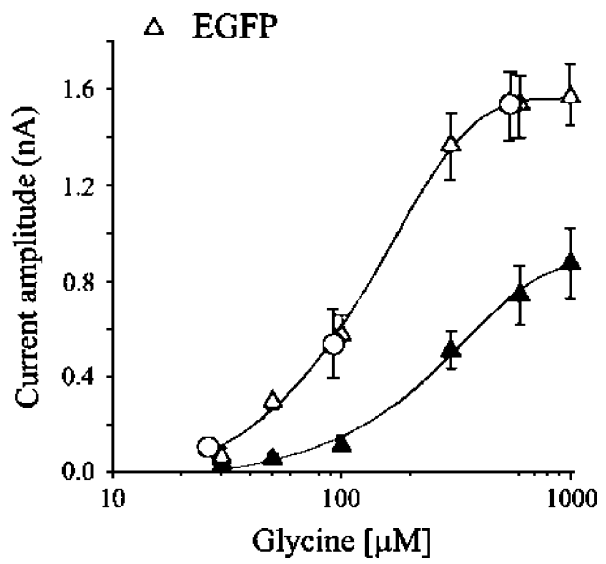

NLS transfected neurons present in the same dish (right). b. Doseresponse curves for glycine-evoked currents obtained in hippocampal neurons transfected with EGFP (open triangles) or with scFvGephyrin-NLS (scFv-Gephyrin, closed triangles). Open circles refer to current responses obtained from nontransfected cells present with scFv-Gephyrin-NLS transfected neurons on the same dish. Each point is the average of 9-20 individual responses

et al. 2004a). Therefore, it would be particularly advantageous to utilize an intrabody instead of iRNA when the active half-life of the target molecule is long, as in the case of neuronal gephyrin. In addition, in the case of proteins with multifunctional domains, intrabodies could be designed to block selectively a particular binding interaction domain therefore leading to the loss of a certain function while sparing others.

Hampering protein function at the posttranslational level may have distinctive advantages, especially when dealing with cells with complex and spatially extended morphology, such as nerve cells, where functional knock-down with intrabodies could be achieved in a more spatially localized manner, or where one could target subcellular pools of a given target protein. The recombinant antibody we selected could be exploited for developing conditional transgenic mice where $\mathrm{scFv}$ expression is under the control of a neuronal cell type specific (tissue-specific) promoter. Alternatively, viral expression vectors may be used to transduce gephyrin-specific intrabodies in nondividing neuronal populations in specific areas of the CNS, giving the possibility to follow the consequences of gephyrin expression and its functional ablation within the same biological preparation. In particular, the occurrence in the same neuronal tissue of cells phenotypically knocked out for gephyrin with WT ones may allow studying the mechanisms regulating neurotransmitter receptor movements on the cell surface and the contribution of receptor clustering to the cross talk between pre and postsynaptic membranes. 
Acknowledgments We are grateful to Beatrice Pastore for cell culture facilities. This work was supported by grants from Ministero Istruzione Universita' e Ricerca (MIUR-PRIN 2005 to EC) and from the European Union (Project 503221 to EC).

\section{Reference}

Andjus, P. R., Stevic-Marinkovic, Z., \& Cherubini, E. (1997). Immunoglobulins from motoneurone disease patients enhance glutamate release from rat hippocampal neurones in culture. Journal of Physiology, 504(Pt 1), 103-112.

Beerli, R. R., \& Barbas, C. F., III (2002). Engineering polydactyl zincfinger transcription factors. Nature Biotechnology, 20, 135-141.

Biocca, S., Neuberger, M. S., \& Cattaneo, A. (1990). Expression and targeting of intracellular antibodies in mammalian cells. EMBO Journal, 9, 101-108.

Birmingham, A., Anderson, E. M., Reynolds, A., Ilsley-Tyree, D., Leake, D., Fedorov, Y., et al. (2006). 3' UTR seed matches, but not overall identity, are associated with RNAi off-targets. Natural Methods, 3, 199-204.

Danglot, L., Rostaing, P., Triller, A., \& Bessis, A. (2004). Morphologically identified glycinergic synapses in the hippocampus. Molecular and Cellular Biochemistry, 27, 394-403.

Feng, G., Tintrup, H., Kirsch, J., Nichol, M. C., Kuhse, J., Betz, H., et al. (1998). Dual requirement for gephyrin in glycine receptor clustering and molybdoenzyme activity. Science, 282, 13211324.

Ito, S., \& Cherubini, E. (1991). Strychnine-sensitive glycine responses of neonatal rat hippocampal neurones. Journal Physiology, 440, $67-83$.

Jackson, A. L., Bartz, S. R., Schelter, J., Kobayashi, S. V., Burchard, J., Mao, M., et al. (2003). Expression profiling reveals off-target gene regulation by RNAi. Natural Biotechnol, 21, 635-637.

Kins, S., Betz, H., \& Kirsch, J. (2000). Collybistin, a newly identified brain-specific GEF, induces submembrane clustering of gephyrin. Natural Neuroscience, 3, 22-29.

Kirsch, J. (2006). Glycinergic transmission. Cell \& Tissue Research, $326,535-540$.

Kirsch, J., \& Betz, H. (1995). The postsynaptic localization of the glycine receptor-associated protein gephyrin is regulated by the cytoskeleton. Journal of Neuroscience, 15, 4148-4156.
Kneussel, M., Brandstatter, J. H., Laube, B., Stahl, S., Muller, U., \& Betz, H. (1999). Loss of postsynaptic GABA (A) receptor clustering in gephyrin-deficient mice. Journal of Neuroscience, 19, 9289-9297.

Kramer, R., \& Cohen, D. (2004). Functional genomics to new drug targets. Naturalist Review of Drug Discovery, 3, 965-972.

Manikandan, J., Pushparaj, P. N., \& Melendez, A. J. (2007). Protein i: interference at protein level by intrabodies. Frontiers in Bioscience, $12,1344-1352$.

Meyer, G., Kirsch, J., Betz, H., \& Langosch, D. (1995). Identification of a gephyrin binding motif on the glycine receptor beta subunit. Neuron, 15, 563-572.

Persic, L., Roberts, A., Wilton, J., Cattaneo, A., Bradbury, A., \& Hoogenboom, H. R. (1997). An integrated vector system for the eukaryotic expression of antibodies or their fragments after selection from phage display libraries. Gene, 187, 9-18.

Saxena, S., Jonsson, Z. O., \& Dutta, A. (2003). Small RNAs with imperfect match to endogenous mRNA repress translation. Implications for off-target activity of small inhibitory RNA in mammalian cells. Journal of Biological Chemistry, 278, 4431244319.

Sola, M., Bavro, V. N., Timmins, J., Franz, T., Ricard-Blum, S., Schoehn, G., et al. (2004). Structural basis of dynamic glycine receptor clustering by gephyrin. EMBO Journal, 23, 2510-2519.

Tian, B., Bevilacqua, P. C., Diegelman-Parente, A., \& Mathews, M. B. (2004). The double-stranded-RNA-binding motif: interference and much more. Nature Reviews Molecular Cell Biology, 5, 1013-1023.

Visintin, M., Meli, G. A., Cannistraci, I., \& Cattaneo, A. (2004a). Intracellular antibodies for proteomics. Journal of Immunological Methods, 290, 135-153.

Visintin, M., Settanni, G., Maritan, A., Graziosi, S., Marks, J. D., \& Cattaneo, A. (2002). The intracellular antibody capture technology (IACT): Towards a consensus sequence for intracellular antibodies. Journal of Molecular Biology, 317, 73-83.

Visintin, M., Tse, E., Axelson, H., Rabbitts, T. H., \& Cattaneo, A. (1999). Selection of antibodies for intracellular function using a two-hybrid in vivo system. Proceedings of the National Academy of Sciences of the United States of America, 96, 11723-11728.

Visintin, M., Quondam, M., \& Cattaneo, A. (2004b). The intracellular antibody capture technology: Towards the high-throughput selection of functional intracellular antibodies for target validation. Methods, 34, 200-214. 